\title{
Clinicopathological and therapeutic features of metaplastic carcinoma of the breast: a study of 15 cases
}

\author{
Hasna Salhi ${ }^{1 *}$, Olfa Jaidane ${ }^{1}$, Olfa El Amine ${ }^{2}$, Med Malek Ayadi', Jamel Ben Hassouna ${ }^{1}$, \\ Tarek Ben Dhieb ${ }^{1}$, Monia Hechiche ${ }^{1}$, Gammoudi Amor ${ }^{2}$, Khaled Rahal ${ }^{1}$
}

${ }^{1}$ Department of Oncological Surgery, ${ }^{2}$ Department of Pathology, Salah Azaiez Institute, Tunis 1006, Tunisia

Received: 21 February 2017

Accepted: 17 June 2017

*Correspondence:

Dr. Hasna Salhi,

E-mail: hasna.salhi@yahoo.fr

Copyright: (C) the author(s), publisher and licensee Medip Academy. This is an open-access article distributed under the terms of the Creative Commons Attribution Non-Commercial License, which permits unrestricted non-commercial use, distribution, and reproduction in any medium, provided the original work is properly cited.

\begin{abstract}
Background: Metaplastic carcinoma of the breast is a rare entity containing a mixture of malignant epithelial and mesenchymal elements. The World Health Organization only recognized it as a distinct pathological entity since 2000. The aim of this study is to better characterize this rare disease.

Methods: We reviewed retrospectively 15 cases of metaplastic carcinoma of the breast treated in our institute between 1994 and 2015. We analyzed clinical, histological, therapeutic and evolutive data.

Results: All patients were females ranging from 29 to 75 years old (median, 50). Tumor size ranged from 20 to 150 $\mathrm{mm}$ (median, $72 \mathrm{~mm}$ ). The Treatment consisted of radical mastectomy in 14 cases, often combined with postoperative radiation and/or chemotherapy. There were 8 cases of carcinosarcoma, 4 cases of spindle cell carcinoma and 3 cases of squamous cell carcinoma. Follow-up data were available on 12 patients. The median follow-up was 47 months (range, 10 to 146 months). Definitive nodal metastases were identified in 6 cases. One patient developed a local recurrence. Extranodal metastases occurred in 3 patients. Three patients died at median interval of 20 months (range, 13-30). Eight patients were alive with no evidence of recurrent or metastatic disease (median, 47 months) and one patient was alive with metastatic disease.

Conclusions: Based on this series, metaplastic breast carcinoma is characterized by a large tumor size at presentation, a low frequency of nodal metastases and a high proportion of triple negativity. Our series is consistent with the literature.
\end{abstract}

Keywords: Breast, Carcinoma, Metaplastic

\section{INTRODUCTION}

Metaplastic Breast Carcinoma (MBC) is a rare subtype of invasive Breast Cancer (BC). It accounts for less than $1 \%$ of all breast carcinomas exhibiting either a mesenchymal or other non-epithelial component or epithelial with squamous differentiation. ${ }^{1}$

This tumor is characterized by a larger size at presentation, lower rates of axillary nodal involvement, higher rates of both local and distant recurrence, higher rates of triple-negative profile (lack of ER, PR and Her2 over expression) as well as sub-optimal response to systemic therapies when compared to other invasive breast cancers.

The World Health Organization (WHO) classified MBC into pure epithelial type and mixed epithelial and mesenchymal type. ${ }^{2}$ 


\section{METHODS}

Patients were selected from the database of the surgical oncology department of Salah Azaiez institute in Tunis between September 1994 and December 2015.

We excluded all patients with mixed histologies, those with a diagnosis of carcinoma in situ and those who had synchronous tumors in another organ.

We obtained and analyzed these clinical parameters: age, tumor size; lymph node status; distant metastasis, surgery performed and adjuvant therapy.

Furthermore, we analyzed all the following pathological variables: hormonal receptors (HRs), the ki-67 proliferation index (with $14 \%$ as a cut-off value) and Her2. Statistically, we analyzed recurrence, progression and survival of the patients through the last follow-up appointment.

Statistical analysis was performed using Statistical Package for the Social Sciences (SPSS) program, version 18.0. OS and DFS rates were estimated by Kaplan-Meier analysis.

\section{RESULTS}

We identified $15 \mathrm{MBC}$ cases. All patients were female, with a median of age 50 years (range, 29 to 79). All the cases were unilateral, ten cases were localized in the left breast and five cases were localized in the right breast. The median tumor size was $72 \mathrm{~mm}$ (range, 20 to 150). There were seven cases classified T2, four cases T3, three cases $\mathrm{T} 4 \mathrm{~b}$ and one case $\mathrm{T} 4 \mathrm{~d}$.

Mammography was performed to all patients. (Figure 1, Figure 2). All masses were highly dense, 8 masses were round, three lobular and four irregulars. Margins were circumscribed in three masses, microlobulated in two cases and indistinct in nine cases. One mass had speculated borders. Calcifications were observed in three cases.

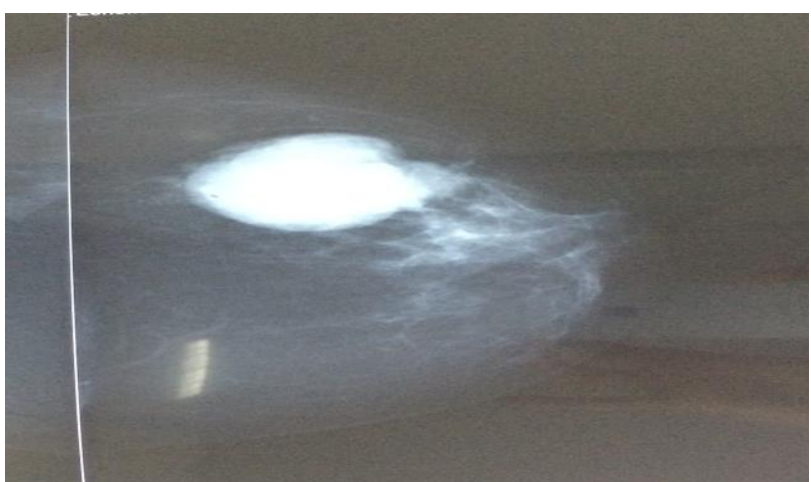

Figure 1: Mammographic appearance of a metaplastic carcinoma in a 53-year-old woman: a circumscribed highly dense mass of the left breast.

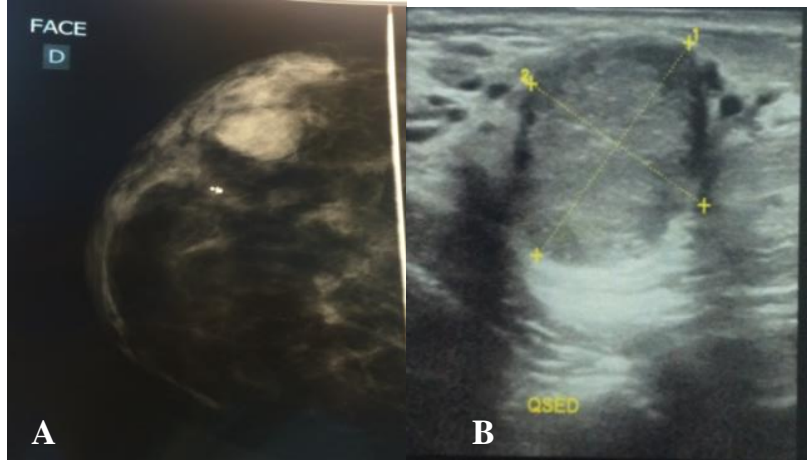

Figure 2: Metaplastic carcinoma of the breast in a 49 year-old woman: Mammography showing a highly dense relatively circumscribed mass in the upperouter quadrant of the right breast (A) and ultrasound showing a hypoechoic solid mass $(28 \mathrm{~mm})$ with long axis perpendicular to the skin (B).

Thirteen patients underwent radical mastectomy and only two patients underwent a conservative surgery consisting of lumpectomy with lymph node dissection. Final histological exam showed: 8 cases of carcinosarcoma, 4 cases of spindle cell carcinoma (Figure 3 ) and 3 cases of squamous cell carcinoma (Figure 4).

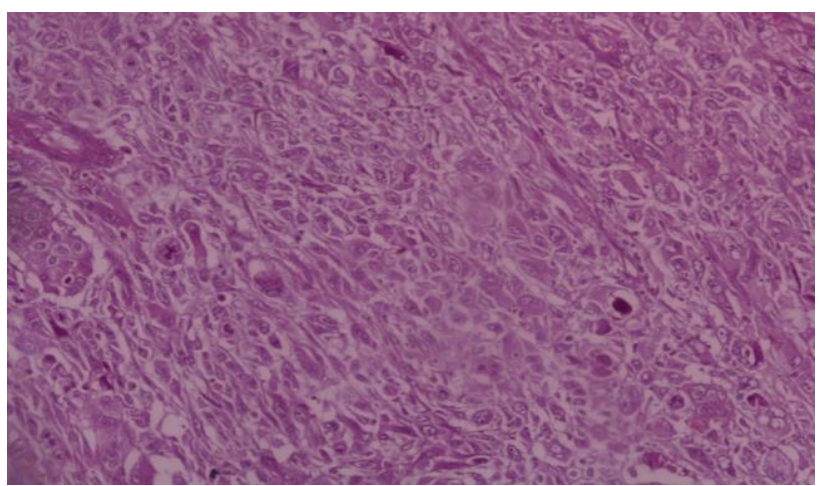

Figure 3: Microscopic examination: tumor composed of malignant spindle cells and atypical epithelial cells

(Hematoxylin-Eosin, high magnification x100).

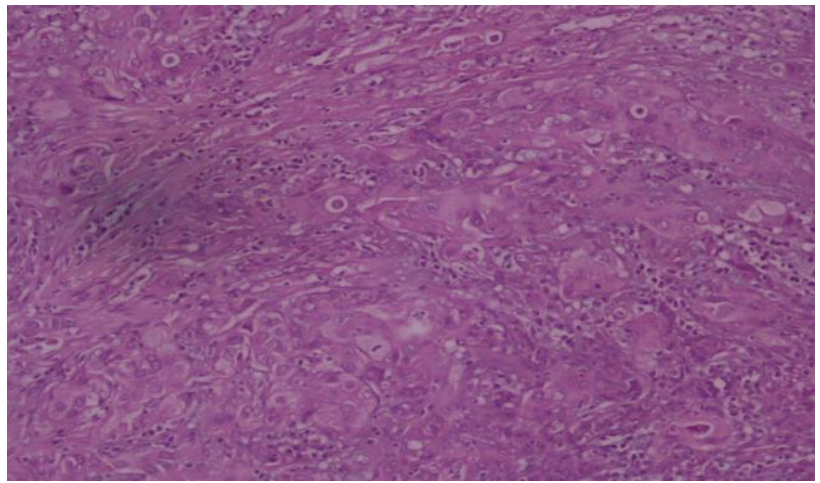

Figure 4: Microscopic examination: tumor cells showing high grade of atypical cell features with presence of squamous differentiation (Hematoxylineosin, high magnification $\mathbf{x 1 0 0}$ ). 
We have noted 6 nodal metastases. Fourteen tumors showed negative hormonal receptors. Her2 was negative in all cases. The ki-67 proliferative index ranges from 12 to $60 \%$, and it was high in 14 cases. (Figure 5).

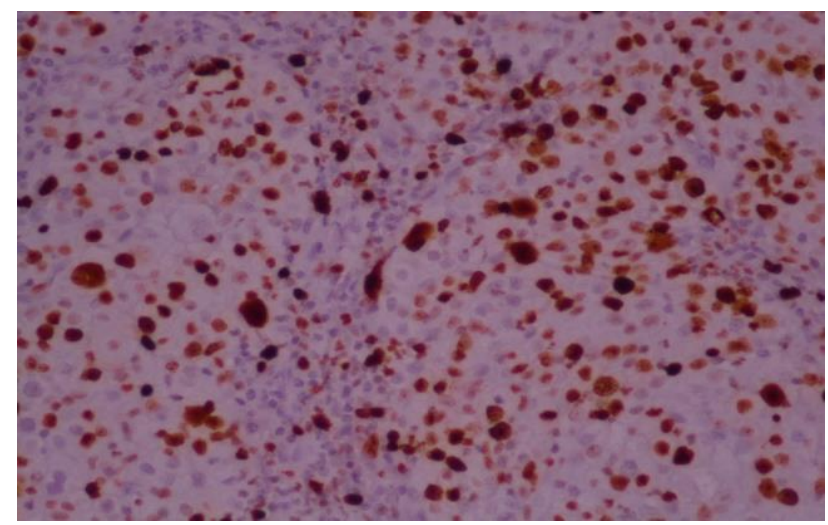

Figure 5: Metaplastic carcinoma with high proliferative index Ki67: $60 \%$ (Immunohistochemistry x 100).

Twelve patients received adjuvant chemotherapy; eleven patients received a FEC100 Fluorouracil+epirubicin+cyclophosphamide)

chemotherapy with taxanes and one patient received adriamycine with 5-FU with endoxan.

Eleven patients received postmastectomy radiation therapy, and two patients had received a radiation therapy after lumpectomy.

Follow-up data were available on 12 of the 15 patients. After a median follow up of 47 months (range: 10 to 146 months), one patient had a loco-regional recurrence (an axillary nodal recurrence). Distant metastases were noted in three patients $(25 \%)$ at a median interval of 15 months (range, 13 to 18 months); in the lung and the liver in two cases, and in the bone and the lung in another case. Three patients died of disease at a median interval of 20 months (range, 13- 30 months). One patient was alive with metastatic disease. Eight patients were alive without evidence of recurrent or metastatic disease. The 5-year OS (overall survival) rate was $60.5 \%$, and the 5-year DFS (Disease Free Survival) was 50.6\%.

\section{DISCUSSION}

Metaplastic carcinoma is a rare malignancy of the breast that accounts less than $1 \%$ of all mammary tumors. ${ }^{1}$

The World Health Organization only recognized MBC as a distinct pathological entity since 2000 .

Most metaplastic carcinomas are sporadic, but they may be a slight propensity for metaplastic spindle cell carcinoma to arise from pre-existing lesions, including papillomas, complex sclerosing lesions and nipple adenomas. $^{2,3}$ Metaplastic carcinomas arising from such lesions can show a varying degree of malignancy, ranging from low to high grade. This type of lesion should also be differentiated from the so-called reactive spindle cell nodule, which is believed to be a benign lesion and may also complicate pre-existing lesions such as papillomas or complex sclerosing lesions. ${ }^{4}$

The WHO classifies MBC into epithelial type and mixed type. ${ }^{5}$ Epithelial-type $\mathrm{MBC}$ is, in turn, classified into squamous cell carcinoma, adenocarcinoma with spindle cell differentiation, and adenosquamous carcinoma. Mixed type $\mathrm{MBC}$ is classified into carcinoma with chondroid metaplasia, carcinoma with osseous metaplasia, and carcinosarcoma. ${ }^{5}$ In our study, there were 8 cases of mixed type (carcinosarcoma), and 7 cases of epithelial type (4 cases of spindle cell carcinoma and 3 cases of squamous cell carcinoma).

Most studies have found that the tumor was large at the time of MBC diagnosis. Pezzi et al. reported that the larger sizes of $\mathrm{MBC}$ at clinical presentation appeared to result from a more rapid growth rate. ${ }^{6} \mathrm{MBC}$ presented with axillary nodal involvement less frequently than did IDC (Invasive Ductal Carcinoma) of the breast. Six patients had nodal involvement in our study (40\%). This is higher than what was observed in previous reports $(6 \%$ to $28 \%)^{7,8}$

Pezzi et al. noted that nodal positivity was more common among the carcinosarcoma variant. ${ }^{6}$ Three of the six patients who had nodal involvement in our study, had a carcinosarcoma variant. There is a high incidence of hormone receptor negativity as well as lower Her-2/neu expression in MBC. ${ }^{9}$

MBCs are often classified along the spectrum of basaltype breast cancers. These tumors represent $75-85 \%$ of all triple negative tumors (estrogen receptor, progesterone receptor, and Her-2 over expression negative). ${ }^{10,11} \mathrm{MBC}$ represents approximately $5 \%$ of triple negative tumors.

In present study, most of the cases showed negative hormone receptors (oestrogen and progesterone receptors). There was only one case of high levels of hormone receptors. This low percentage of expression of the hormone receptors in our study $(6.66 \%)$ is in agreement with the $0-25 \%$ reported in the literature. ${ }^{12,13}$

Expression of Her2 was also law (we have not noted any case of over-expression of Her2). Metaplastic breast tumors do tend to express the HER1/EGFR (epidermal growth factor) receptor at a considerably higher rate than most other types of breast carcinoma, and this is a somewhat unique histological identifier for this type of breast cancer, and may lead to some new and potentially beneficial treatment strategies.

Song et al., comparing a group of 55 patients with MBC with a group of 767 age-matched patients with IDC, noted that over-expression of Ki-67 was more common in 
the MBC group compared with the IDC group (Ki-67 $\geq 14 \%, \quad 87 \%$ versus $63 \%, \quad \mathrm{P}=0.001) .{ }^{14}$ The surgical treatment of $\mathrm{MBC}$ has largely paralleled that of IDC. Relative to those with IDC, a higher percentage of patients with $\mathrm{MBC}$ receive mastectomy rather than lumpectomy. ${ }^{6-15}$ In our study $86.7 \%$ of the patients had a radical mastectomy.

Despite the large tumor size at presentation, MBC histology should not preclude breast conservation therapy in appropriate patients. Tseng and Martinez found no difference in overall or disease-specific survival whether MBC patients were treated with mastectomy or lumpectomy, even after controlling for known prognostic factors. ${ }^{16}$ Similarly, Dave et al. found no difference in overall or disease-free survival between patients with MBC undergoing either modified radical mastectomy or breast conservation therapy. ${ }^{17}$ Compared to stagematched women with IDC, those with MBC receiving adjuvant chemotherapy have poor survival. Single institution retrospective studies suggest that these tumors are largely chemoresistant. ${ }^{18}$
This resistance to chemotherapies is likely a product of the complex genetic and nongenetic mechanisms within $\mathrm{MBC}$ that result in phenotypically diverse subclones and intratumoral heterogeneity. Information regarding the role of adjuvant radiation therapy (RT) for the treatment of MBC is sparse. Dave et al. reported a $10.5 \%$ rate of local recurrence for patients receiving lumpectomy and adjuvant radiation. ${ }^{17}$

Postmastectomy RT has a more limited role. In this setting, RT is recommended to patients with four or more metastatic axillary nodes, large $(>$ or $=5 \mathrm{~cm}$ ) primary tumors and chest wall invasion [19-20]. Tseng et al. described mastectomy patients who had received RT demonstrating a 33\% decreased risk of death from any cause. Patients undergoing mastectomy with tumors <or= $5 \mathrm{~cm}$ and less than 4 metastasis axillary lymph nodes, however, derived no benefit from RT. ${ }^{16}$ We have recapitulated the results of treatment in our study compared to related studies in Table 1.

Table1: Comparison of current study with related studies.

\begin{tabular}{|c|c|c|c|c|c|}
\hline Study & Age (years) & TN\% & Type of surgery & СТ\% & RT\% \\
\hline Song et al. ${ }^{13}$ & $\begin{array}{l}<50: 49,1 \\
>=50: 50,1\end{array}$ & 67,3 & $\begin{array}{l}\text { MRM 92,7 } \\
\text { BSC 7,7 }\end{array}$ & 48 & 49,1 \\
\hline Nelson et al. ${ }^{21}$ & 61 & 77,8 & $\begin{array}{l}\text { MRM } 53 \\
\text { BSC } 47\end{array}$ & NA & 48,3 \\
\hline Barquet et al. ${ }^{22}$ & 49,6 & 83,6 & $\begin{array}{l}\text { MRM 91,7 } \\
\text { BSC } 8,3\end{array}$ & $\begin{array}{l}\text { ind CT 79,2 } \\
\text { adj CT 39,6 }\end{array}$ & 47,9 \\
\hline Current study & 50 & 93,3 & $\begin{array}{l}\text { MRM 86,7 } \\
\text { BSC 13,3 }\end{array}$ & $\operatorname{adj}$ CT 80 & 86,6 \\
\hline
\end{tabular}

Recent studies have investigated receptors that may potentially serve as novel targets for chemotherapy regimens. One such target is EGFR (Her-1). Leibl and Moinfar found that targeted protein kinase such as gefitinib might be effective for some patients with MBC. ${ }^{23}$ Although molecular analysis for possible genetic alterations in the EGFR might be required, there is a good possibility that women fighting MBC might benefit from treatment with protein kinase inhibitors, such as gefitinib and cetuximab. The prognosis of $\mathrm{MBC}$, with respect to 'triple negative' status, is contrary to the norm. Recent studies have shown, contrary to a generally accepted view, that a non-triple-negative metaplastic breast carcinoma actually has a poorer prognosis when compared with the triple-negative metaplastic breast carcinoma. ${ }^{18}$

\section{CONCLUSION}

MCB is an infrequent entity and thus is rarely studied. It had distinct clinicopathological features, which include a large tumor size at presentation, a low proportion of lymph node metastasis, a high proportion of triple negativity and a high ki67 proliferation index. Poor prognostic indicators for $\mathrm{MBC}$ include a tumor size larger than $5 \mathrm{~cm}$, lymph node involvement and $\mathrm{Ki}-67>$ or $=$ $14 \%$.

Due to its rarity and heterogeneity, there is no "standard" therapy for all patients with MBC. Surgical treatment and axillary staging parallel that of IDC with breast conservation therapy are appropriate for a select group of patients. Targeted therapies based on individualized gene profiling are promising for the future but are not commonly utilized. Adjuvant radiation should be considered part of the multimodality therapy for patients with MBC, regardless of the type of surgery.

Funding: No funding sources

Conflict of interest: None declared

Ethical approval: The study was approved by the Institutional Ethics Committee 


\section{REFERENCES}

1. Tavassoli FA. Classification of metaplastic carcinomas of the breast. Pathol Annu. 1992;27:89119

2. Gobbi H, Simpson J F, Jensen R A. et al Metaplastic spindle cell breast tumours arising within papillomas, complex sclerosing lesions, and nipple adenomas. Mod Pathol. 2003;16:893-901.

3. Denley H, Pinder SE, Tan PH. Metaplastic carcinoma of the breast arising within complex sclerosing lesion: a report of five cases. Histopathol. 20003;6:203-9.

4. Gobbi H, Tse G, Page DL. Reactive spindle cell nodules of the breast after core biopsy or fine-needle aspiration. Am J Clin Pathol. 2000;113:288-294.

5. Tavassoli FA, Devilee P. World Health Organization classification of tumors: tumors of the breast and female genital organs. Pathology and genetics of tumors of the digestive system. In World Health Organization Classification of Tumors. Lyon, France: IARC Press;2003:37-41.

6. Pezzi CM, Patel-Parekh L, Cole K, Franko J, Klimberg VS, Bland K. Characteristics and treatment of metaplastic breast cancer: analysis of 892 cases from the national cancer data base. Annal Surg Oncol. 2007;14(1):166-73.

7. Wargotz ES, Norris HJ. Metaplastic carcinomas of the breast. I. Matrix-producing carcinoma. Hum Pathol. 1989;20:628-35.

8. Wargotz ES, Norris HJ. Metaplastic carcinomas of the breast. III. Carcinosarcoma. Cancer. 1989;64:1490-9.

9. Bae SY, Lee SK, Koo MY. The prognoses of metaplastic breast cancer patients compared to those of triple-negative breast cancer patients. Breast Cancer Res Treatm. 2011;126(2):471-8.

10. Sørlie T. Molecular portraits of breast cancer: tumour subtypes as distinct disease entities. European J Cancer. 2004;40(18):2667-75.

11. Carey LA, Dees EC, Sawyer L. The triple negative paradox: primary tumor chemosensitivity of breast cancer subtypes. Clin Cancer Res. 2007;13(8):232934.

12. Barnes PJ, Boutilier R, Chiasson D, Rayson D. Metaplastic breast carcinoma: clinical-pathologic characteristics and HER2/neu expression. Breast Cancer Research and Treatment. 2005;91(2):173-8.

13. Poggi MM, Danforth DN, Sciuto LC. Eighteen-year results in the treatment of early breast carcinoma with mastectomy versus breast conservation therapy: the National Cancer Institute randomized trial. Cancer. 2003;98(4):697-702.

14. Song Y, Liu X, Zhang G, Song H, et al., Unique clinicopathological features of metaplastic breast carcinoma compared with invasive ductal carcinoma and poor prognostic indicators. World J Surg Oncol. 2013;11:129.

15. Gibson GR, Qian D, Ku JK, Lai LL. Metaplastic breast cancer: clinical features and outcomes. Am Surg. 2005;71(9):725-30.

16. Tseng WH, Martinez SR. Metaplastic breast cancer: to radiate or not to radiate? Annal Surg Oncol. 2011;18(1):94-103.

17. Dave G, Cosmatos H, Do T, Lodin K, Varshney D. Metaplastic carcinoma of the breast: a retrospective review. Internat $\mathbf{J}$ Radiat Oncol Biol Physics. 2006;64(3):771-5.

18. Hennessy BT, Giordano S, Broglio K. Biphasic metaplastic sarcomatoid carcinoma of the breast. Annals Oncol. 2006;17(4):605-613.

19. Ragaz J, Jackson SM. Adjuvant radiotherapy and chemotherapy in node-positive premenopausal women with breast cancer. New Engl J Med. 1997;337(14):956-962.

20. Fowble B, Gray R, Gilchrist K, Goodman RL, Taylor S, Tormey DC. Identification of a subgroup of patients with breast cancer and histologically positive axillary nodes receiving adjuvant chemotherapy who may benefit from postoperative radiotherapy. J Clin Oncol. 1988; 6(7):1107-17.

21. Nelson RA, Guye ML, Luu T, Lai LL. Survival outcomes of metaplastic breast cancer patients: results from a US population-based analysis. Ann Surg Oncol. 2015;22(1):24-31

22. Barquet-Munoz SA. Villarreal-Colin SP. HerreraMontalvo LA. Metaplastic breast cancer: a comparison between the most common histologies with poor immunohistochemistry factors. BMC Cancer. 2015;15:75

23. Leibl S, Moinfar F. Metaplastic breast carcinomas are negative for Her-2 but frequently express EGFR (Her-1): potential relevance to adjuvant treatment with EGFR tyrosine kinase inhibitors? J Clin Pathol. 2005;58(7):700-4

Cite this article as: Salhi $\mathrm{H}$, Jaidane $\mathrm{O}$, Amine OE, Ayadi MM, Hassouna JB, Dhieb TB, et al. Clinicopathological and therapeutic features of metaplastic carcinoma of the breast: a study of 15 cases. Int J Reprod Contracept Obstet Gynecol 2017;6:3219-23. 